\title{
I pericoli di un metodo a-logico
}

\section{Anna Foa}

Storicamente, 3 (2007).

ISSN: 1825-411X. Art. no. 21. DOI: 10.12977/stor546

La domanda da cui parte il libro di Ariel Toaff, Pasque di sangue, è una domanda a cui, dalla fine dell'Ottocento in qua, la storiografia aveva rinunciato, considerandola legata alla polemica antigiudaica e poi antisemita, e quindi superata da ogni seria ricerca scientifica: le accuse di omicidio rituale che costellano la storia degli ebrei europei dopo il XII secolo riflettevano, sia pur parzialmente e solo in alcuni casi, fatti realmente accaduti, o erano costruzioni mitologiche inventate dai persecutori? Insomma, gli ebrei avevano davvero l'abitudine di uccidere a Pesach bambini cristiani per fabbricare azzime o a scopo medicinale? Così, se alla fine dell'Ottocento, più esattamente nel 1891, Hermann Strack poteva ancora nel suo libro (Das Blut im Glauben und Aberglauben der Mensheit) sentire il bisogno di affermare a chiare lettere l'innocenza degli ebrei, successivamente la storiografia aveva preso a dare assolutamente per scontato queste premesse ad indagare invece la costruzione del mito del sangue nella storia. Nessuno storico degno di questo nome aveva finora preso sul serio la domanda se gli ebrei potessero in qualche modo avere praticato sacrifici rituali. Nemmeno quanti avevano indagato sull'ostilità anticristiana del mondo ebraico, come Cecil Roth negli anni '30 del Novecento e recentemente Elliot Horowitz a proposito del Purim, o Yisrael Yuval a proposito del qiddush ha shem nella Germania delle Crociate, si erano posti la domanda di una possibile realtà dell'accusa di omicidio rituale, lavorando invece sul terreno delle rappresentazioni e del loro reciproco influsso tra i due mondi. 
Ed ecco che il libro di Toaff riporta la questione della realtà sul tappeto e lo fa partendo da un punto di vista del tutto inedito; quello della necessità di sfuggire ad un'ottica puramente negativa, in chiave antisemita, degli ebrei, e di ridar loro carne e sangue, azioni e percezioni, passando da una mera storia dell'antisemitismo ad una storia in positivo degli ebrei. Un ottimo proposito, che non mi pare però del tutto nuovo, come dimostra, per l' Italia, la storiografia più recente (e come non citare a questo proposito i contributi apparsi nella rivista «Zakhor», diretta dallo stesso Ariel Toaff e arrivata al suo decimo anno?)

Peccato però che questo proposito di restituire realtà agli ebrei, nel caso di un'accusa antiebraica come quella del sangue, finisca per tradursi nel risultato di prender per buone le confessioni degli accusati, e attribuire loro una effettiva realtà storica; "dobbiamo chiederci se le confessioni degli imputati siano resoconti puntuali di eventi effettivamente accaduti, oppure di credenze, da inquadrarsi in contesti simbolici, mitici e magici da ricostruire. ... Sciogliere i nodi non è compito agevole né semplice, ma forse neppure impossibile» (9). In sostanza, se gli ebrei confessano, e se gli ebrei nella storia sono attori, e non oggetti passivi, allora dobbiamo cercare il vero che c'è nelle loro confessioni. Di qui, parte il percorso di Toaff, volto ad «indagare sull'eventuale presenza di credenze ebraiche negli omicidi rituali, legati alla celebrazione della Pasqua».

Ma di che area geografica stiamo parlando e di che periodo storico? L'area su cui il discorso di Toaff si muove è quella coperta dalla presenza di ebrei ashkenaziti; la Germania, I'Italia settentrionale in primo luogo. E qui, nell'evidente e precostituita volontà di contrapporre un ebraismo ashkenazita violento e «fondamentalista» ad un civile e illuminato ebraismo italiano e sefardita, Toaff trascura il fatto che accuse di omicidio rituale si ritrovano anche in area a presenza sefardita, in Spagna, in Italia meridionale, e nei testi antiebraici spagnoli, come in Alonzo de Espina.

In realtà, Toaff postula che in generale nell'area ashkenazita si verificassero casi di omicidio ritualizzato di bambini cristiani, anche se non si esprime 
direttamente su questo punto e parla invece, nell'introduzione, di «usi e tradizioni legati a esperienze irripetibili», di un fenomeno «limitato a gruppi presso i quali tradizioni popolari che nel tempo avevano aggirato o sostituito le norme rituali della halakhah ebraica e consuetudini radicate, impregnate di elementi magici e alchemici, si sposavano in un micidiale cocktail con un fondamentalismo religioso violento e aggressivo» (13).

Ma di che gruppi stiamo parlando? In realtà, infatti, questi piccoli gruppi fondamentalisti non appaiono nel libro, e invece il suo discorso si concentra sulla comunità di Trento, ashkenazita sì ma non fondamentalista. L'impressione del lettore è che si stia parlando di un clima generale e non di casi limitati e particolari. Forse perché, se così avesse fatto, Toaff si sarebbe trovato nell'obbligo di documentarli, di trovare dei riscontri. II che non ha fatto. Altrettanto complesso è il discorso sulla periodizzazione, che copre esplicitamente un periodo di quattro secoli, un po' troppo per parlare di pochi casi isolati, e volta a suggerire l'idea di pratiche di lungo periodo. II libro, in ogni caso, non si concentra su alcuni casi di gruppi «fondamentalisti», per usare l'espressione anacronistica e fuori luogo dell'autore, ma sul caso di Trento (perché l'unico ben documentato), e di lì, per analogia, sugli altri casi famosi di accuse del sangue: Endingen, Ratisbona, Rinn, Portobuffolé, e via discorrendo. In sostanza, tempi e spazi appaiono dilatati rispetto all'assunzione originaria, mai seriamente approfondita, di piccole sette fondamentaliste.

Al cuore delle argomentazioni di Toaff è, lo abbiamo detto, il processo di Trento. È la sua analisi, l'analisi delle deposizioni processuali degli ebrei accusati di aver ucciso ritualmente a Pesach il piccolo Simonino per mescolarne il sangue alle azzime, che lo spinge a considerare che le deposizioni dei rei confessi non potevano essere soltanto un'invenzione dei giudici. Di lì, il suo percorso di ricerca si dipana, per successivi avvicinamenti fondati soprattutto sull'associazione, attraverso l'uso medicinale del sangue, le percezioni anticristiane sottese al Purim, il rituale della circoncisione, il seder di Pesach, fino all'asserzione, espressa talvolta come mera possibilità, talaltra come plausibilità talaltra come fatto concreto, della realtà 
dell'omicidio rituale e del commercio di sangue di bambino cristiano, trasferito da un luogo all'altro nelle sacche dei mercanti ebrei, con tanto di certificato di kasherutt.

Ma questi capitoli di considerazioni antropologiche sul sangue e la centralità del sangue nel mondo ebraico non reggerebbero da soli la costruzione di Toaff, e sono da lui presentati soltanto come un contorno, un riscontro a quella che è la sua tesi fondamentale, su cui basa la sua rilettura dei processi: che le risultanze dei processi siano troppo concordanti e troppo particolareggiate per essere solo invenzione dei giudici. Che esse riflettano quindi non un immaginario condiviso da parte degli inquisiti (ipotesi meritevole di una discussione seria), ma dati di realtà. In questa sua analisi, Toaff considera come riscontri il fatto che gli inquisiti nominassero persone realmente esistenti, descrivessero situazioni reali nello spazio e nel tempo, parlassero un ebraico-tedesco che i giudici non potevano conoscere, come non potevano conoscere i particolari dei rituali ebraici.

È questo, degli elementi reali riscontrabili nelle confessioni rese sotto tortura in questi secoli, un argomento già proprio, fin dall'inizio, dei giudici. Nel 1631 , in un testo famoso destinato a combattere i processi contro le streghe, la Cautio criminalis, così ne parlava il gesuita tedesco Friedrich von Spee, confessore e difensore delle donne accusate di stregoneria:

Dall'esperienza sembrerebbe che siano vere quelle dichiarazioni rese sotto tortura che concordano con le circostanze: se, ad esempio, Sempronia ammette sotto tortura di aver ucciso, attraverso arti magiche, tre mesi prima, la mucca di Tizio, come pure due anni prima il bambino di Gracco, e altre cose simili, e i giudici verificano e scoprono che effettivamente tre mesi prima la vacca di Tizio era morta, e davvero, anche, il figlio di Gracco era morto da due anni, ecc., che cosa è più evidente della veridicità di quelle dichiarazioni rese sotto tortura? Questo è esattamente quello che è accaduto; dunque sono veritiere le dichiarazioni rese sotto tortura. Così ragiona il volgo, e non solo quello, ma perfino molti giudici esperti, inquisitori, consiglieri dei principi, che spesso ho ascoltato; e sono rimasto sbalordito, perché non stavano dicendo per scherzo o per il gusto di discutere, come in un primo momento avevo creduto, ma seriamente e con la ferma convinzione di aver dimostrato con 
prove inoppugnabili che la confessione di Sempronia doveva essere stata assolutamente vera.

Rispondo. È gravissimo errore pensare di aver fornito, in questo modo, una dimostrazione sicura e sentirsene soddisfatti. Ecco, infatti, come stanno le cose: Sempronia non poteva ignorare ciò che è noto a tutto il villaggio, finanche ai bambini, e cioè che, in quel luogo e in quelle date, la vacca era morta, il bambino aveva perso la vita e altre vicende simili che erano accadute nel villaggio. Quando dunque le sofferenze della tortura la spingevano a confessare di aver commesso qualche maleficio, allora ammetteva quelle cose che sapeva essere accadute, attribuendosene la responsabilità [1].

Le procedure della Germania di Spee non erano certo molto distanti da quelle della Trento in cui si svolse il processo contro gli ebrei nel 1475. Ed è vero, come Toaff si premura di sottolineare, che l'uso della tortura era regolamentato, non selvaggio. E allora? Forse che una tortura regolamentata, decisa su decreto, era meno dolorosa? Molto potremmo citare, anche tratto dallo stesso testo di Spee, su tale regolamentazione. Ma basti qui dire ciò che Toaff non dice nel suo libro, che essa veniva stabilita anche nel caso di discordanze fra le deposizioni dei vari inquisiti, nelle denunce discordanti dei complici, e in genere qualora senza tortura (de plano ) gli inquisiti avessero ritrattato la loro precedente deposizione resa sotto tortura (la ragione di questa ultima pratica è che si veniva a creare una contraddizione tra due testimonianze dello stesso imputato, contraddizione che poteva essere risolta solo attraverso una nuova tortura). In sostanza, per smettere di essere torturati, gli imputati dovevano non soltanto confessare, ma confessare cose plausibili, coerenti con le altre confessioni, passibili di riscontri. Ecco quindi che le confessioni si riempiono di particolari, di nomi, di date, di fatti. Tutte cose che gli inquisitori non potevano conoscere, un quadro però che rendeva plausibile e reale la confessione dell'omicidio, la sua descrizione particolareggiata. Senza contare che il processo condotto dal vescovo Hinderbach a Trento si svolse nella più netta 
illegalità, come sostenne il visitatore mandato da Roma, il vescovo Battista de' Giudici. A meno di non voler prendere per buona, come sembra fare Toaff, l'idea che il vescovo fosse stato corrotto dagli ebrei, come denunciò allora Hinderbach. Ma dobbiamo abbracciare davvero acriticamente tutte, proprio tutte, le tesi del vescovo di Trento?

Consideriamo, per entrare un po' di più nel merito delle sue argomentazioni, il XII capitolo intitolato /I memoriale della passione, in cui Toaff affronta il problema cruciale per la sua tesi dell'«uso del sangue d'infante cristiano nella celebrazione della Pasqua ebraica». Esso, scrive Toaff all'inizio del capitolo, «era apparentemente oggetto di una normativa minuziosa, per lo meno a quanto risulta dalle deposizioni di tutti gli imputati ai processi di Trento» (173): una frase in cui la parola chiave non è «almeno a quanto risulta», cioè un limitativo dell'affermazione generale, ma quel «tutti» che sottolinea l'omogeneità del quadro.

«Ogni eventualità era prevista e affrontata - prosegue scivolando a dare consistenza e realtà al quadro - e, quasi facesse parte integrante delle più collaudate regole del rito, l'impiego del sangue era sottoposto ad una casistica ampia ed esauriente» (173). Da quel momento in poi, Toaff prosegue attraverso una serie di descrizioni minuziose del modo in cui gli ebrei avrebbero mescolato il sangue di un bambino cristiano alle azzime pasquali, tutte desunte dai documenti processuali, almeno fino alla nota 8, dove a corroborare la confessione di uno degli imputati, Angelo da Verona (confessione che secondo Toaff fornisce una presentazione «molto colorita e credibile» del rito) si introduce il testo settecentesco del Bonelli, violentemente antigiudaico. Alla nota 10, invece, viene introdotto il testo apologetico di Divina, Storia del beato Simone da Trento, del 1902, a sostenere l'affermazione, emersa in tutte le confessioni, che il capofamiglia scioglieva nel rito il sangue nel vino solo prima di recitare le maledizioni d'Egitto. Ma il capitolo prosegue con le rivelazioni di un personaggio particolarmente interessante e centrale nel testo di Toaff, Israel Wolfgang, un giovane pittore convertito, poi anch'egli accusato di aver partecipato all'uccisione di Simonino e giustiziato con gli altri. Le sue «rivelazioni», come 
le chiama Toaff, sono tratte dal solito Divina, e corroborate da altre deposizioni processuali. A questo punto, Toaff interviene direttamente, con effetto teatrale, a dare la sua interpretazione: a raccontare nei particolari lo svolgimento di un Seder potevano essere solo gli ebrei, i giudici non erano in grado di suggerirglielo. Ergo, «ipotizzare che fossero proprio loro a dettare con le torture quelle rappresentazioni del rituale del Seder, con le relative formule liturgiche in ebraico, appare poco credibile» (178). II compito di procurarsi il sangue dell'infante cristiano, insiste Toaff, incombeva al capofamiglia. Fra le note a supporto di questa tesi, oltre alle solite deposizioni processuali, studi ebraici sul ruolo dei capifamiglia ashkenaziti non nelle uccisioni rituali ma più banalmente... nella celebrazione dei riti di Pasqua. In tutto questo ragionamento, senza troppo parere, è avvenuto il passaggio dalla celebrazione dei riti pasquali al compito di procurarsi il sangue di un bambino cristiano. Ma, sono sempre le deposizioni processuali a dirlo, se un capofamiglia era povero, era dispensato dal procurarsi un ingrediente costoso come quello. E Divina viene nuovamente citato a sostegno della tesi che gli ebrei ricchi fossero soliti distribuire generosamente sangue di bambino agli ebrei poveri. E a questo punto, scivolando ancora in un deciso indicativo, Toaff ci da la sua spiegazione di un rito la cui esistenza non ha in nessun modo provato: «ll rito del vino, o del sangue, e delle maledizioni aveva una doppia valenza. Da una parte doveva ricordare la miracolosa salvezza di Israele...Dall'altra si proponeva di avvicinare la redenzione finale, preparata dalla vendetta di Dio sui gentili...» (181). Ma no, subito dopo apprendiamo che la spiegazione non è una interpretazione di Toaff, bensì una deposizione processuale di Trento, quella del vecchio Mosè di Wurzburg. Gli ebrei inquisiti a Trento sono infatti pronti a rispondere alle domande dei loro giudici sul significato e l'origine di quel rito, anche se non sono in grado di fornire delle risposte precise, limitandosi a richiamarsi alla tradizione talmudica e a sottolineare la trasmissione solo orale di questi rituali, per mantenerne il segreto. Tutti gli ebrei processati inoltre insistono sul fatto che tutta la comunità doveva partecipare all'infanticidio rituale, una deposizione che a noi sembra fatta apposta per 
coinvolgere collettivamente gli ebrei ma che Toaff, senza spiegarne il nesso ma solo in una postmoderna assonanza, collega... agli usi funebri collettivi dell'ebraismo tedesco. E di nuovo torna qui la contrapposizione fra ashkenaziti ed italiani. I giudici infatti chiedono come mai gli ebrei italiani non conoscessero queste usanze, e gli inquisiti affermano trattarsi di un'usanza del solo mondo tedesco.

Ma non di tutto, afferma Toaff. Infatti, in una deposizione trentina si affermava che due viaggiatori ebrei tedeschi si erano rifiutati di far commercio per loro di sangue cristiano. Segno, afferma Toaff, prendendo immediatamente per buona, testualmente, la deposizione, che non tutti gli ashkenaziti compivano questi riti, ma solo "circoli fondamentalisti dell'ortodossia ashkenazita» (186). E a riprova cita il Ristretto della vita e martirio di S. Simone fanciullo di Trento, opera agiografica composta nel 1594 a Roma. Perché, conclude Toaff, il rito doveva essere mantenuto segreto. Oltre ai passaggi in questo senso dei soliti imputati che deponevano sotto tortura, Toaff si appoggia infine a altri testi diversi da quelli agiografici: Le Toledot leshu, testi di polemica anticristiana databili tra il IV e I'VIII secolo, diffusi nel mondo ebraico (ma naturalmente Toaff sottolinea soltanto la diffusione in area ashkenazita), in cui si ritrova frequente il richiamo al segreto. Nulla nei testi citati può far supporre che quel segreto si riferisca ai sacrifici di bambini cristiani e non invece alle storie anticristiane, che il mondo cristiano considerava gravemente blasfeme. Ma per Toaff il cerchio si è chiuso, e l'uso del sangue è provato.

Lo stesso procedimento a-logico ritroviamo nel resto del libro. Facciamo ancora un esempio, quello di un capitolo centrale per le tesi di Toaff, il capitolo 6, sul Sangue magico e terapeutico. Anche questo capitolo prende le mosse dalle deposizioni processuali, che rivelano l'uso di sangue di bambini cristiani a scopo medicinale, e in particolare per guarire la ferita della circoncisione. Ma gli ebrei, scrive, non menzionano il sangue fra i rimedi della circoncisione. Omissione che «potrebbe non essere casuale» (95), e che spinge Toaff a vedere se i convertiti, sempre pronti a rivelare i 
«segreti» degli ebrei, lo citino. Eppure, neanche i convertiti ne parlano. «Si potrebbe dedurre ... che sia una chimera tendenziosa invenzione degli inquisitori... Ma questa sarebbe una risposta sbagliata e fuorviante» (97). Tutto qui. A supportare questa incredibile affermazione, Toaff usa quelli che chiama i testi della Kabbalah pratica. Testi tutti molto più tardi e che non parlano di sangue cristiano ma di sangue in generale, e che possono al massimo dimostrare la diffusione del sangue ad uso medicinale. È pur vero che Toaff accosta all'uso medicinale del sangue il costume superstizioso di mangiare il prepuzio dopo la circoncisione, che non c'entra niente, a rigor di logica, ma che contribuisce a dare degli ebrei un'immagine di cannibalismo che non stona nel quadro d'insieme. Su questa base, Toaff torna indietro nel tempo, a criticare la deposizione difensiva a Trento del vecchio Mose da Wurzburg, che si richiamava al divieto biblico del sangue, fuorviante secondo Toaff perchè l'uso di sangue (animale) era invece previsto dalle raccolte di segullot in uso presso gli ashkenaziti. E qui introduce, con un piccolo inciso, «umano o animale», il sangue umano, che fino ad allora non c'era. Di nuovo, dopo essersi dilungato sull'uso del sangue di coniglio, introduce il sangue di bambino cristiano, traendolo non dai testi ebraici bensì, ancora una volta, dalle deposizioni processuali (102). A questo punto, Toaff spiega la contraddizione tra norma (che vieterebbe l'uso del sangue) ed uso (che invece lo consentirebbe), risolvendola, attraverso la distinzione tra sangue dei cristiani, permesso e sangue degli ebrei, proibito, distinzione fatta da Lazzaro da Serravalle, imputato a Trento, e dal solito convertito Israel Wolfgang (la fonte su cui si basa, alle note 40 e 42, è sempre Bonelli). Poi Toaff passa alla contraddizione fra minhag e norma, per arrivare in fondo, quadrando il cerchio, a dire che 
Dagli atti del processo di Trento emergerebbe non soltanto l'uso generalizzato di sangue da parte degli ebrei tedeschi per scopi curativi e magici, ma anche la necessità che essi, a detta degli inquisitori, avrebbero sentito di provvedersi di sangue cristiano (e di un bambino battezzato in particolare) soprattutto per celebrare i riti di Pesach (107). 
Subito dopo si introduce il commercio del sangue di bambino con tanto di certificato di kasherut: "A detta degli accusatori di Trento, la clientela più accorta aveva preteso che i rivenditori si provvedessero di certificati di idoneità rituale» (108). Ma, "per quanto paradossale e inverosimile possa sembrare ai nostri occhi questa circostanza ... crediamo che essa meriti una certa attenzione». Senza supporto e senza riscontri. II testo termina suggerendo che nei certificati di kasherutt del sangue del bambino sacrificato, «la scritta omettesse intenzionalmente ogni avviso esplicito al tipo di mercanzia trattata» (109). Ancora una volta, il cerchio si chiude, e Toaff da per dimostrato il suo assunto di partenza, che gli ebrei si procurassero il sangue di bambini cristiani e lo vendessero con un certificato di kasherut.

Ancora una parola sul linguaggio di Toaff: uno stile piacevolissimo, immaginifico, coloristico, pieno di frasi ad effetto, che inganna e trascina il lettore.Vengono in mente i libri di Piero Camporesi, un autore molto amato e citato da Toaff, con i suoi materiali affastellati e con il suo procedere per assonanze e somiglianze, lasciando libertà alle parole. L'apparato di note è amplissimo, tale da suggerire al lettore non specialista l'idea di un supporto filologico di primo piano, di un libro insomma documentatissimo. Invece non lo è, ma di questo possono accorgersene solo gli specialisti, facendo un attento raffronto fra ogni affermazione e la nota su cui si appoggia. Un procedimento, quello usato in questo libro da Toaff, che uno storico non dovrebbe assolutamente usare e che fa di questo libro una specie di "falso": non semplicemente un libro che presenta un'interpretazione sbagliata, ma un libro che manipola le fonti.

La ricaduta del lancio scandalistico del libro da parte di Sergio Luzzatto e del suo successivo ritiro da parte di Ariel Toaff merita inoltre qualche riflessione immediata. Temo però che sarà questo un tema su cui bisognerà riflettere ancora in futuro. Dopo il ritiro da parte di Toaff del libro, e dopo che le critiche degli storici, tutte documentate e tutte basate su una accurata lettura da parte loro del libro, sono state fatte passare per una campagna di 
linciaggio preventivo, il discorso sui media si è spostato: non si parla più della colpevolezza o meno degli ebrei medioevali, ma della lobby ebraica che è tanto potente da impedire ad un libro di circolare e che opera preventive scomuniche. In questo senso, il comunicato di condanna della consulta rabbinica, sia pur moderato nei toni e lontano dall'essere una scomunica, è stato, a mio avviso, un errore gravissimo. L'idea che circola ora è che dove c'è fumo c'è arrosto, e dove gli ebrei vogliono togliere dalla circolazione un libro, vuol dire che si tratta di un libro che presenta verità sgradite. È chiaro che simili voci non possono trovare risposata a livello razionale. Siamo di fronte al riemergere di vecchie mitologie che pensavamo sepolte e che evidentemente poco basta a far riapparire. Personalmente, io auspico che il libro ritorni in circolazione. Ha fatto ormai, a livello di opinione comune, tutto il danno che poteva fare, meglio che sia sugli scaffali di tutte le librerie e che affronti le critiche degli studiosi. Per questo, ho deciso di regalare alla Biblioteca del mio Dipartimento la mia copia di Pasque di sangue.

\section{Note}

* Anna Foa è professore di Storia moderna presso l'Università "La Sapienza" di Roma. Specialista di storia sociale e culturale e di storia degli ebrei, è autrice tra gli altri del volume sugli Ebrei in Europa dalla Peste Nera all'emancipazione (Roma-Bari, Laterza, 1998, trad. ingl. California University Press, 2000).

[1] F. von Spee, I processi contro le streghe. Cautio Criminalis, a cura di A. Foa, Roma, Salerno, 2004, 177-78. 